\title{
Mathematical evaluation of entrance surface dose (ESD) for patients examined by diagnostic $x$-rays
}

\begin{abstract}
Exposures from diagnostic medical X-rays are the most important synthetic source of exposure to ionizing radiation in several countries. However information on medical exposure of diagnostic X-rays is integrated into international legislative repertories. In Sebha city, there is a lack of data on the assessment of patient's entrance surface dose (ESD) and the health risk from conventional radiography in daily routine of diagnostic medical examinations. In this research, the Entrance Surface Dose (ESD) was estimated for adult patients underwent diagnosis X-ray examinations in one of radiographic center in Sebha city. The ESD has been estimated indirectly using exposure factors for patents. The results showed that the mean patient entrance surface doses (ESD) were $41.73 \pm 5.84 \mathrm{mGy}, 7.43 \pm 2.58 \mathrm{mGy}, 103.7 \pm 125.53 \mathrm{mGy}, 7.25 \pm 4.32$ $\mathrm{mGy}$ and $11.24 \pm 16.18 \mathrm{mGy}$ respectively for Pelvis (AP), Chest (AP), Lumbar Spine (AP), Cervical Spine (AP) and Skull (AP). The mean ESD values are found to be higher than mean ESD reference values. This indicates that the necessity for reducing the patient doses to the acceptable levels recommended by the international radiation protection commissions to protect patients from risk of higher exposures to diagnostic X-rays.
\end{abstract}

Keywords: entrance surface dose (ESD), diagnostic x-rays, lumbar spine, cervical spine
Volume I Issue | - 2017

\author{
Abubaker Alghoul, Mustafa Mohamed \\ Abdalla, Hamed Mohamed Abubaker \\ Department of Physics, University of Sebha, Libya
}

Correspondence: Abubaker Alghoul, Department of Physics, Faculty of Science, University of Sebha, Libya,Tel 00218695 7760,Email alghoul75@yahoo.com

Received: June 05, 2017 | Published: July 06, 2017
Abbreviations: ESD, entrance surface dose; ICRP, international committee of radiation protection; TLD, thermoluminesent dosimeters; FSD, focus to surface distance

\section{Introduction}

X-ray examinations are an established tool of medical diagnosis. Patients undeniably benefit from these examinations; even though the ionizing nature of X-rays means that there are unavoidable effects which may definitely occur. For example the deleterious effects for patients happed while patients overexposure to X-rays. For this reason, it is necessary to apply radiation protection principles in medical diagnosis using X-rays procedures. In addition, all exposures from X-rays must be justified and optimized in terms of benefit and risk. ${ }^{1-3}$ It also well-known that the X-rays transfer a certain amount of energy to biological system as it is penetrating; however this energy may be risky of cells those building up biological entities. ${ }^{4}$ There is increased concern about exposing patients from higher levels of diagnostic X-rays. This concern noticed in recommendations of the International Committee of Radiation Protection (ICRP). ${ }^{5-7}$

The recommendations directed to put together all countries to provide restricted radiation dose for patients undergoing the X-ray examinations in the almost all radiographic centers and radiology departments at hospitals. One of the major important factors in radiation protection is patient dose. The patient dose is usually specified by means of determining entrance surface dose (ESD) for patient being exposed to diagnostic X-rays. The entrance surface dose (ESD) is defined as the absorbed dose to air at the point of intersection of the X-ray beam axis with the entrance surface of the patient, including backscatter. ${ }^{8}$ The entrance surface dose is one of the basic quantities for measuring the patient dose and as well for optimizing the patient dose. This quantity is basic criterion for comparing to the other international reference dose levels which is very important from the point of view for radiation protection. ${ }^{9}$ There are many different methods for determination of the ESD. In common, the ESD can be determined mainly by two methods: either by direct measurements using Thermoluminesent dosimeters (TLD) stacked on the patient's skin or indirectly via mathematical model calculations based on the X-ray machine output. The ESD can be determined from the measurements performed on a phantom and data from the patient's exploration. ${ }^{10-12}$

Generally, applying the TLD in measuring the ESD for patients involves time consuming and using special equipments which may not be available at the most radiographic centers. On the other hand, using ionization chambers to measure the ESD for patients require using of conversion factors to convert the ionization chamber (IC) reading to absorbed dose which to some extent is complicated method. ${ }^{13}$ Although due to the hard accessibility of TLD or IC instruments, the mathematical method was employed in this study to estimate the ESDs. The aim of this research was to estimate the ESD for patients exposed to diagnostic X-Rays at medical radiographic centers in Sebha city. To fulfill this objective The ESD was estimated applying Chuan and Tsai formula for many patients exposed to diagnostic X-rays for six examinations. The values obtained of the ESD from this research were compared with the international ESD values reported in the literature.

\section{Materials and methods}

The X-ray diagnostic equipment was used to expose patients with total number of 45 patients. The specification of this machine complies with the DHHS requirements, minimum inherent filtration Aluminum equivalent $2 \mathrm{~mm} \mathrm{Al} / 75$, X-ray rating up to $150 \mathrm{kv}_{\mathrm{p}}$. At the beginning, the patient's data such as Age and Gender was recorded and then the patient was centered by technician to be ready for radiographic. The parameters such as peak tube voltage $\left(\mathrm{kv}_{\mathrm{p}}\right)$, exposure current and time product $(\mathrm{mAs})$ and focus to surface distance (FSD) were recorded at 
the time of the examination. This information was recorded for each patient undergoing the particular diagnostic procedure. In present work the Chuan and Tsai formula ${ }^{14}$ is applied to calculate the ESD for patients coming to the X-ray radiographic center. The entire selected sample was mainly from adults: men, women and including few cases of children. This formula is given as follows:

$$
\operatorname{ESD}(\mathrm{mGy})=\mathrm{c}\left(\frac{\mathrm{kv} \mathrm{p}_{\mathrm{p}}}{\mathrm{FSD}}\right)^{2}\left(\frac{\mathrm{mAs}}{\mathrm{mm} \cdot \mathrm{Al}}\right)
$$

Where $\mathrm{kv}_{\mathrm{p}}$ represents $\mathrm{X}$-ray peak tube voltage and $\mathrm{mAs}$ represents the exposure value which means that tube's current times exposure time. While FSD (Focus to Skin Distance) represents the measured distance between X-ray tube and patient part being exposed to X-rays, $\mathrm{mm}$. Al gives minimum inherent filtration Aluminum equivalent and $\mathrm{c}$ is constant which equals to 0.2775 . The obtained data was analyzed using Excel.

\section{Results}

This work was carried out in one of radiographic center in Sebha city. One X-ray unit was included in this study. The results obtained were recorded in tables shown below. The results included gender, patient's age, $\mathrm{kv}_{\mathrm{p}}$, mAs, FSD and entrance surface dose (ESD) for all different cases being exposed to diagnostic X-rays. The results also included medical procedures involving different positioning of patients such as Anterior-Posterior (AP) and Lateral (LAT). The other medical procedures including Posterior-Anterior (PA) are not considered in this study owning to the little cases being examined. Tables listed underneath (1-6) indicate the estimated values of the ESD in every examination. Table 1 indicates exposure factors and estimated ESDs for patients undergone to Pelvis (AP) examination. Table 2 indicates exposure factors and the estimated ESDs for patients undergone to Chest (AP) examination. While Table 3 shows the exposure factors and estimated ESDs for patients undergone to Lumbar Spine (AP) examination. Table 4 shows also the exposure factors and estimated ESDs for patients undergone to Cervical Spine examination. Tables $5 \& 6$ present the exposure factors and estimated ESDs for patients undergone to Skull (AP \& LAT) examinations. Table 7 indicates the mean values of the patient exposure factors, such as $\mathrm{kv}_{\mathrm{p}}, \mathrm{mAs}, \mathrm{FSD}$ and ESD. The last Table 8 shows the compression between the results obtained during this study, previous studies and the established international reference levels of ESDs.

Table I The Estimated ESDS for Pelvis

\begin{tabular}{lllllll}
\hline Number & Gender & Age & Diagnostic type & $\mathbf{K v}_{\mathrm{p}}$ & Mas & FSD $(\mathbf{C m})$ \\
\hline I & F & 4 I & 88 & 79 & 43 \\
2 & F & 44 & 88 & 79 & 43 \\
3 & F & 63 & 80 & 66 & 43 \\
4 & F & 38 & 84 & 79 & 43 \\
s5 & F & 24 & 85 & 79 & 43 \\
6 & M & 39 & & 89 & 79 & 43 \\
7 & F & 30 & Pelvis AP & 80 & 66 & 43 \\
8 & F & 31 & & 86 & 79 & 43 \\
9 & F & 29 & 86 & 79 & 43 \\
10 & F & 24 & 85 & 79 & 43 \\
\hline
\end{tabular}

Table 2 The Estimated ESDS for Chest

\begin{tabular}{llllllll}
\hline Number & Gender & Age & Diagnostic type & $\mathbf{K v}_{\mathrm{p}}$ & Mas & FSD(Cm) & ESD (Mgy) \\
\hline I & F & 24 & 80 & 66 & 100 & 5.86 \\
2 & $\mathrm{~F}$ & 80 & 121 & 12 & 53 & 8.68 \\
3 & $\mathrm{~F}$ & 70 & 123 & 10 & 53 & 7.47 \\
4 & $\mathrm{~F}$ & 52 & 120 & 12 & 45 & 11.84 \\
5 & $\mathrm{~F}$ & 58 & 85 & 79 & 100 & 7.92 \\
6 & $\mathrm{~F}$ & 64 & 75 & 66 & 100 & 5.15 \\
7 & $\mathrm{~F}$ & 60 & & 80 & 79 & 100 & 7.01 \\
8 & $\mathrm{~F}$ & 46 & Chest AP & 85 & 79 & 100 & 7.92 \\
9 & $\mathrm{~F}$ & 60 & & 85 & 79 & 100 & 7.92 \\
I0 & $\mathrm{M}$ & 24 & 70 & 66 & 100 & 4.49 \\
II & $\mathrm{F}$ & 24 & 90 & 79 & 100 & 8.88 \\
12 & $\mathrm{M}$ & 4 & 50 & 45 & 100 & 1.56 \\
\hline
\end{tabular}


Table 3 The Estimated ESDS For Lumbar Spine

\begin{tabular}{llllllll}
\hline Number & Gender & Age & Diagnostic type & $\mathbf{K v}_{\mathrm{p}}$ & Mas & FSD(Cm) & ESD (Mgy) \\
\hline I & F & 40 & & 90 & 450 & 43 & 273.52 \\
2 & $\mathrm{~F}$ & 38 & 110 & 245 & 43 & 222.46 \\
3 & $\mathrm{~F}$ & 43 & & 90 & 132 & 43 & 80.23 \\
4 & $\mathrm{~F}$ & 42 & & 104 & 197 & 53 & 105.25 \\
5 & $\mathrm{~F}$ & 63 & Lumbar Spine & 125 & 590 & 53 & 455.36 \\
6 & $\mathrm{~F}$ & 26 & AP & 106 & 284 & 53 & 157.62 \\
7 & $\mathrm{~F}$ & 63 & & 90 & 123 & 43 & 74.76 \\
8 & $\mathrm{M}$ & 19 & & 90 & 123 & 43 & 74.76 \\
9 & $\mathrm{M}$ & 55 & & 122 & 284 & 43 & 317.2 \\
10 & $\mathrm{M}$ & 39 & & 110 & 245 & 43 & 222.46 \\
\hline
\end{tabular}

Table 4 The Estimated ESDS for Cervical Spine

\begin{tabular}{llllllll}
\hline Number & Gender & Age & Diagnostic type & $\mathbf{K v}_{\mathrm{p}}$ & Mas & FSD(Cm) & ESD (Mgy) \\
\hline I & F & 35 & & 85 & 79 & 118 & 5.69 \\
2 & F & 40 & & 70 & 66 & 117 & 3.28 \\
3 & 26 & & 71 & 66 & 53 & 16.43 \\
4 & F & Cervical Spine & 88 & 79 & 100 & 8.49 \\
5 & F & 32 & LAT & 80 & 66 & 100 & 5.86 \\
6 & F & 33 & & 80 & 66 & 100 & 5.86 \\
7 & F & 36 & & 75 & 66 & 100 & 5.15 \\
\hline
\end{tabular}

Table 5 The Estimated ESDS for Skull

\begin{tabular}{llllllll}
\hline Number & Gender & Age & Diagnostic type & $\mathbf{K v}_{\mathbf{p}}$ & Mas & FSD(Cm) & ESD Mgy \\
\hline I & M & 4 & & 66 & 45 & 86 & 3.68 \\
2 & F & 43 & Skull AP & 81 & 79 & 45 & 35.51 \\
3 & F & 5 & & 65 & 55 & 100 & 3.22 \\
4 & M & I & & 58 & 55 & 100 & 2.57 \\
\hline
\end{tabular}

Table 6 The Estimated ESDS for Skull

\begin{tabular}{llllllll}
\hline Number & Gender & Age & Diagnostic type & $\mathbf{K v}_{\mathrm{p}}$ & Mas & FSD(Cm) & ESD Mgy \\
\hline I & F & 8 & & 70 & 66 & 156 & 1.84 \\
2 & F & 4 & Skull LAT & 70 & 66 & 100 & 4.49 \\
3 & M & 5 & & 70 & 66 & 100 & 4.49 \\
\hline
\end{tabular}

Table 7 Mean Values of the Estimated ESDS

\begin{tabular}{|c|c|c|c|c|c|c|}
\hline & This Study & 15 & 16 & 17 & 18 & 19 \\
\hline Diag.Type & ESD mGy & ESD mGy & ESD $\mathrm{mGy}$ & ESD mGy & ESD mGy & ESD mGy \\
\hline Pelvis (AP) & 41.73 & $*$ & 0.62 & 1.25 & 10 & 4 \\
\hline Chest (AP) & 7.43 & $*$ & 0.18 & $*$ & $*$ & $*$ \\
\hline Lumbar Spine (AP) & 103.7 & 22.61 & 2.25 & 10 & 6 & 10 \\
\hline Cervical Spin(LAT) & 7.25 & 2.4 & $*$ & $*$ & $*$ & $*$ \\
\hline Skull (AP) & 11.24 & $*$ & $*$ & $*$ & $*$ & $*$ \\
\hline
\end{tabular}


Table 8 Comparison between the Present Study and the Literature

\begin{tabular}{lllll}
\hline Diag. type & $\mathbf{k v}_{\mathrm{p}}$ & $\mathbf{m A s}$ & FSD & ESD $(\mathbf{m G y})$ \\
\hline Pelvis & $80-89$ & $66-79$ & 43 & $31.70-46.96$ \\
AP & -85.1 & -76.4 & -43 & $(41.73 \pm 5.84)$ \\
Chest & $50-123$ & Oct-79 & $45-100$ & $1.56-11.84$ \\
AP & -81.85 & -51.69 & -80 & $(7.43 \pm 2.58)$ \\
Lumbar & $90-125$ & $123-590$ & $43-53$ & $74.76-455.36$ \\
Spine & -103.7 & -267.3 & -46 & $(103.7 \pm 125.53)$ \\
Cervical Spine & $70-88$ & $66-79$ & $53-118$ & $3.28-16.43$ \\
LAT & -78.43 & -69.71 & -98.28 & $(7.25 \pm 4.32)$ \\
Skull & $58-81$ & $55-79$ & $45-100$ & $3.22-35.51$ \\
AP & -67.5 & -58.5 & -82.75 & $(11.24 \pm 16.18)$ \\
\hline
\end{tabular}

\section{Discussion}

This study was directed to the estimate entrance surface dose (ESD) for patients underwent diagnostic X-ray examinations at radiology department. The selected sample in this investigation included 45 patients. The data collected from one center in Sebha city. The protocol used to image patients in this center was the highest kv and the lowest mAs for Pelvis (AP), Chest (AP), Cervical Spine (AP) and Skull (AP). Although the lowest $\mathrm{kv}_{\mathrm{p}}$, the highest $\mathrm{mAs}$ were used to image Lumbar Spine (AP). The estimated ESDs varied from 31.7046.96mGy for Pelvis (AP), 1.56-11.84mGy for Chest (AP), 74.76455.36mGy for Lumbar Spine (AP), 3.28-16.43mGy for Lumbar Cervical (AP) and 3.22-35.51mGy for Skull (AP). When comparing the values obtained of ESDs from this study to previous studies and international reference levels (IRLs), (Table 8), the mean ESDs evaluated by this work were found to be higher than both the previous studies and the IRLs. The minimum dose value $(7.25 \mathrm{mGy})$ in this study was with case of Cervical Spine (LAT). While the maximum dose value (103.7mGy) in this study was with case of Lumbar Spine (AP). In all cases of examinations, higher values of the ESDs are obtained. Judging from Table 8, the ESD values are more than 10times larger than those reported in the literatures. That is due to the higher loading of the exposure rate (mAs), in addition to the insufficient competency of technicians. It is significance to radiation protection and image quality to decreases the risks for patients. Increasing the competency of technologists through training courses helps to avoid repeating the dose given for patients, necessitate of continuity of such as these studies to cover all radiology centers and hospitals in Sebha city. ${ }^{15-19}$

\section{Acknowledgements}

None.

\section{Conflict of interest}

Author declares that there is no conflict of interest.

\section{References}

1. Olivera Ciraj, Srpko Markovic, Dusko Kosutic. Patient Dosimetry in Diagnostic Radiology. Nuclear Technology and Radiation Protection. 2003;36(1):1-6.

2. International Commission on Radiology Protection. 1990 Recommendations of the ICRP. Canada: Publication (60) Annals ICRP 21 Pergamon Press; 1991.

3. Olivera Ciraj, Srpko Marković, Duško Košutić. Patient Dose from Conventional Diagnostic Radiology Procedures in Serbia and Montenegro. The Journal of Preventive Medicine. 2004;12(4):26-34.

4. Alghoul A, Yasir M. Alternative Mathematical Form for Determining the Effectiveness of High-LET Radiations at Lower Doses Region. International Journal of Radiology and Imaging Technology. 2016;2(1):1-4.

5. ICRP. Avoidance of Radiation Injuries from Interventional Procedures. International Commission on Radiation Protection ICRP Publication 85. Ann ICRP. 2000;30(2).

6. WHO. Quality Assurance in Diagnostic Radiology. Geneva, Switzerland: World Health Organization; 1982.

7. CEC Radiation Protection 33 EUR 9728EN. Luxembourg: Office for Official of the EURO Pean Community; 1985.

8. Code of Safe Practice for the Use of X-Rays in Medical Diagnosis. New Zealand: National Radiation Laboratory Ministry of Health 1994: 1-88.

9. International Commission on Radiation Units and Measurements. Radiation Dosimetry: X-rays Generated at Potentials of 5 to $150 \mathrm{Kv}$ 17ICRU, USA; 1970

10. Jornet N. Determination of Entrance Surface Dose in Standard Explorations in Radio diagnostic. New York. 2013.

11. Gaetano Compagnone, Laura Pagan, Carlo Bergamini. Comparison of Six Phantoms for Entrance Skin Dose Evaluation in 11 Standard X-ray Examinations. Journal of Applied Clinical Medical Physics. 2005;6(1):101-113.

12. Shabon M. Pediatric X-ray Examinations Special Review. Journal of Nuclear and Radiation Physics. 2013;82(1):4-35.

13. Ding C, LB Li, JP Wang, et al. Medical Radiation Usage and Exposures from Medical Radiation X-ray Diagnostic in Shandong Province of China. Radiation Protection Dosimetry. 2001;93(2):261-266.

14. Tung CJ, Tsai HY. Evaluation of Gonad Dose for Diagnostic Radiology. Proc Natl Sci Counc Repub China B. 1999;23(3):107-113.

15. Yousif A. Estimation of Entrance Surface Dose (ESD) of Patients Examined by Diagnostic X-Ray at Sebha Medical Center. Journal of Applied Science. 2016.

16. Jumaa Yousif Tamboul, Mohamed Yousef, Khadija Mokhtar, et al. Assessment of Entrance Surface Dose for the Patients from Common Radiology Examinations in Sudan. Life Science Journal. 2014;11(2):1-5.

17. Diagnostic Reference Levels in Medical Imaging, review and additional Advice. A web module produced by committee 3 of the international commission on radiological protection (ICRP). 1996.

18. Hull, East Yorkshire. Hospitals Radiation Protection Service (Reference Value Proposed as DRL To be considered in 2005).

19. Quality Criteria Reference Doses [EUR96], NA. 